\title{
Exposure Sponsor Defined Identifier
}

National Cancer Institute

\section{Source}

National Cancer Institute. Exposure Sponsor Defined Identifier. NCI Thesaurus. Code C83287.

One or more sponsor defined characters used to name an exposure. 